\title{
Prise en charge des infarctus cérébraux
}

\section{Treatment of Acute Ischaemic Stroke}

\author{
S. Deltour \\ Reçu le 22 mars 2018; accepté le 18 juin 2018 \\ (C) SRLF et Lavoisier SAS 2018
}

Résumé La prise en charge de l'infarctus cérébral aigu s'est considérablement améliorée ces dernières années grâce à de nouvelles techniques combinant imagerie et nouveaux traitements. La formalisation d'un parcours spécifique, multidisciplinaire (neurologues, radiologues, urgentistes et réanimateurs), a permis un véritable gain d'efficacité. Une course contre la montre s'enclenche dès les premiers signes cliniques évocateurs d'un accident vasculaire cérébral. L'approche thérapeutique actuelle, combinant thrombectomie et fibrinolyse dans les six premières heures après l'apparition des symptômes, a transformé le pronostic. Très récemment, des études rapportent un bénéfice de la thrombectomie jusqu'à 24 heures du début des symptômes chez des patients très sélectionnés. En outre, l'accès rapide à une unité neurovasculaire (UNV) est déterminant dans la réduction de la morbidité et du handicap, en dehors de tout traitement spécifique de recanalisation (effet stroke center). Ces nouvelles données impactent considérablement le schéma organisationnel des premières heures, définissant deux stratégies d'orientation du patient : transfert direct (mother ship) dans un centre associant UNV et centre de neuroradiologie interventionnelle (NRI) ou accueil dans une UNV et transfert secondaire dans un centre de NRI si nécessaire (drip and ship).

Mots clés AVC · Unités neurovasculaires · Thrombolyse · Thrombectomie

\begin{abstract}
The management of acute cerebral infarct has considerably changed in recent years, thanks to new techniques combining imaging and new treatments. The formalization of a specific multidisciplinary course ( emergency physicians, neurologists, radiologists and resuscitators) allowed a real gain of efficiency. A real race against the clock starts at the first clinical signs suggestive of stroke. Rapid
\end{abstract}

\footnotetext{
S. Deltour $(\varangle)$

Service des urgences cérébrovasculaires, groupe hospitalier Pitié-Salpêtrière,

47-83, boulevard de 1'Hôpital, F-75013 Paris, France

e-mail : sandrine.deltour@aphp.fr
}

access to a NeuroVascular Unit (UNV) is crucial in the prognosis, allowing to reduce morbidity and disability, apart from any specific recanalization treatment (Stroke Center effect). IV thrombolysis with alteplase administered within $4 \mathrm{~h} 30 \mathrm{~min}$ of cerebral infarction remained until 2015, the only specific reference treatment. Since then, mechanical thrombectomy within $6 \mathrm{~h}$ must be associated with proximal occlusion. These new data have a significant impact on the early hours of organizational planning, defining two patient referral strategies: direct transfer (Mother Ship) in a UNVCenter for Interventional NeuroRadiology (NRI) or reception in a UNV and secondary transfer in a NRI center, if necessary (Drip and Ship). Very recently, studies report the benefit of thrombectomy up to $24 \mathrm{~h}$ from the onset of symptoms in highly selected patients. These data remain to be confirmed.

Keywords Stroke $\cdot$ Stroke units $\cdot$ Thrombolysis · Thrombectomy

\section{Structures hospitalières spécialisés}

\section{Définition-organisation}

Les premières unités neurovasculaires (UNV) sont apparues dans les années 1970. Elles ont été ensuite organisées par la publication de trois circulaires ministérielles [1-3]. Ces UNV, dédiées à la prise en charge de patients victimes d'accident vasculaire cérébral (AVC), sont composées d'un personnel spécialisé et bénéficiant d'une formation spécifique régulière : médecins, infirmiers, aides-soignants, kinésithérapeutes, ergothérapeutes, orthophonistes, psychologues et assistantes sociales. Les UNV comprennent des lits d'hospitalisation et de soins intensifs, en lien avec un service de neuroradiologie. Ces structures ont comme mission de permettre un accueil des patients 24 heures sur 24,7 jours sur 7 . Elles constituent l'épine dorsale de la filière de la prise en charge des AVC depuis le préhospitalier jusqu'au retour au domicile du patient. 


\section{Bénéfice des UNV}

Le bénéfice pour les patients admis dans les UNV n'est plus à démontrer : la spécificité de la prise en charge en UNV améliore le pronostic des patients victimes d'AVC, non seulement en diminuant la mortalité, mais aussi en réduisant la probabilité d'un handicap et le risque d'institutionnalisation en dehors de tout geste de revascularisation. La revue Cochrane portant sur 31 essais randomisés (6 936 patients) a évalué un parcours de soins impliquant une UNV à une prise en charge en hospitalisation conventionnelle [4]. Les trois critères de jugement étudiés étaient le décès, la dépendance et l'institutionnalisation à la fin de la période de suivi (médiane $=1$ an). Pour tous les critères choisis, les résultats étaient significativement en faveur d'un parcours incluant une UNV : réduction significative du risque de décès de $18 \%$ (odds ratio [OR] : 0,82; intervalle de confiance [IC] $95 \%$ : $[0,73-0,92] ; p=0,001)$, de décès ou d'institutionnalisation de $19 \%$ (OR : 0,81; IC $95 \%$ : [0,74-0,90] ; $p<0,0001)$ et de décès ou de dépendance de $21 \%$ (OR : $0,79$; IC $95 \%$ : $[0,71-0,88] ; p<0,0001)$. De plus, la mortalité diminuait sans augmenter le nombre de patients dépendants. Il est important de noter que la majorité des études incluses dans cette revue Cochrane ont été réalisées avant l'avènement $\mathrm{du}$ traitement par thrombolyse. Ces données ont été confirmées "dans la vraie vie » par l'analyse de cohortes de patients : ces études observationnelles montraient que la prise en charge en UNV réduisait significativement le risque relatif de décès de $21 \%(\mathrm{OR}: 0,79$; IC $95 \%$ : $[0,73-0,86])$ et de décès ou de dépendance de $23 \%$ (OR : $0,87$ : IC $95 \%:[0,80-0,95])$ à un an de l'AVC [5].

\section{Un réseau régional de soins gradués}

Ainsi, tout patient victime d'un AVC devrait avoir accès à une UNV et entrer dans un parcours de soins animé et organisé à partir de cette unité spécialisée. En effet, nous n'avons aucune preuve dans la littérature qui permettrait d'exercer un triage et d'exclure des patients sur la base de l'âge avancé ou de la sévérité de l'AVC. Nous disposons actuellement de trois types de structures définies selon l'intégration au sein de l'établissement hospitalier : une UNV, un centre de neuroradiologie interventionnelle (NRI) ou de service de neurochirurgie $(\mathrm{NCH})$.

- les établissements ayant une UNV : «UNV de territoire » ou «UNV de proximité » (134 centres recensés en France en 2017);

- les établissements ayant une UNV et disposant de services de NRI (37 centres recensés en France en 2017) et de $\mathrm{NCH}$ pour la prise en charge des patients nécessitant une expertise spécifique avec des actes hautement spécialisés de NRI (notamment la thrombectomie) et de $\mathrm{NCH}$ : «UNV de recours »;

- les établissements recevant des urgences, n'ayant pas d'UNV, mais disposant d'une équipe de médecins formés, ayant un accès direct à l'IRM ou à défaut au scanner, une liaison télémédecine avec l'UNV et des protocoles et de procédures permettant d'assurer un accueil précoce des patients souffrant d'un AVC, de préciser le diagnostic et d'organiser la prise en charge du patient, avec un éventuel transfert en UNV.

\section{Thrombolyse intraveineuse}

\section{Efficacité de l'altéplase}

La thrombolyse intraveineuse (IV) par altéplase (rt-PA) a été jusqu'en 2015 le seul traitement spécifique de prise en charge aiguë dans les infarctus cérébraux (IC). Son administration est constituée par un bolus (10\% de la dose) suivi d'une perfusion continue d'une heure.

Son efficacité dans les premières trois heures de l'IC a été démontrée dans l'étude NINDS (National Institute of Neurological Disorders and Stroke) [6]. L'étude ECASS III (European Cooperative Acute Stroke Study III) a ensuite démontré une efficacité jusqu'à 4 heures 30 minutes après le début des symptômes [7]. L'analyse combinée de ces deux études et d'autres études ayant évalué des délais plus larges a confirmé l'efficacité du rt-PA IV [8]. En France, le traitement a obtenu l'autorisation de mise sur le marché (AMM) en 2002 pour la fenêtre $0-3$ heures après le début des symptômes et en 2012 pour la fenêtre 3-4,5 heures. Le critère principal de ces études est fondé sur le score de Rankin modifié à 90 jours (mini-Rankin score ou mRS) qui évalue la dépendance du patient. Il va de 0 à 6 . Aucun symptôme : score à 0 . Pas d'incapacité en dehors des symptômes : activités et autonomie conservées : score à 1 . Handicap faible : incapable d'assurer les activités habituelles mais autonomie : score à 2. Handicap modéré : besoin d'aide (non autonome), mais marche possible sans assistance : score à 3 . Handicap modérément sévère : marche et gestes quotidiens impossibles sans aide : score à 4 . Handicap majeur : alitement permanent, incontinence et soins de nursing permanents : score à 5 . Décès : score à 6 .

Dans l'étude NINDS, le traitement thrombolytique diminuait le risque de décès ou de dépendance de $10 \%$ à trois mois (l'aspirine était dix fois moins efficace) sans effet sur la mortalité ( $21 \%$ dans le groupe placebo versus $17 \%$ dans le groupe avec fibrinolytique) [6]. Dans l'étude ECASS III, 821 patients ont été randomisés pour recevoir un traitement entre 3 heures et 4 heures 30 minutes après les premiers symptômes par rt-PA $(0,9 \mathrm{mg} / \mathrm{kg})$ ou par placebo. À 90 jours, 
la proportion de patients ayant un pronostic favorable (mRS à 0 ou 1) était significativement supérieure dans le groupe avec rt-PA : $52,4 \%$ dans le groupe rt-PA versus $45,2 \%$ dans le groupe placebo, soit un bénéfice absolu de 7,2\% $(\mathrm{RR}=1,16$; IC $95 \%:[1,01-1,34] ; p=0,04)$. La mortalité était identique dans les deux groupes (rt-PA : 7,7\% ; placebo : $8,4 \%$ ) [7].

La synthèse de toutes ces études montre très clairement que le bénéfice du traitement diminue drastiquement avec l'intervalle de temps qui sépare la prise en charge du début des symptômes (Fig. 1). En d'autres termes, même si les résultats de l'étude ECASS III ont permis d'élargir la fenêtre thérapeutique, l'objectif doit rester de traiter le plus rapidement possible une fois que le diagnostic est établi. Ainsi, une thrombolyse réalisée dans les 90 minutes permet de diminuer de $50 \%$ le handicap fonctionnel à trois mois. Il est à noter également qu'il existe toujours un bénéfice pour les patients thrombolysés de plus de 80 ans [8].

La faisabilité d'administrer ce traitement spécialisé en pratique courante a été apportée par un très large registre européen, le registre SITS-MOST [9]. La mortalité, la proportion de patients ayant un score de mRS à 0 ou 1 et le taux d'hémorragies intracérébrales symptomatiques étaient tout à fait comparables à ce qui avait été observé dans les essais

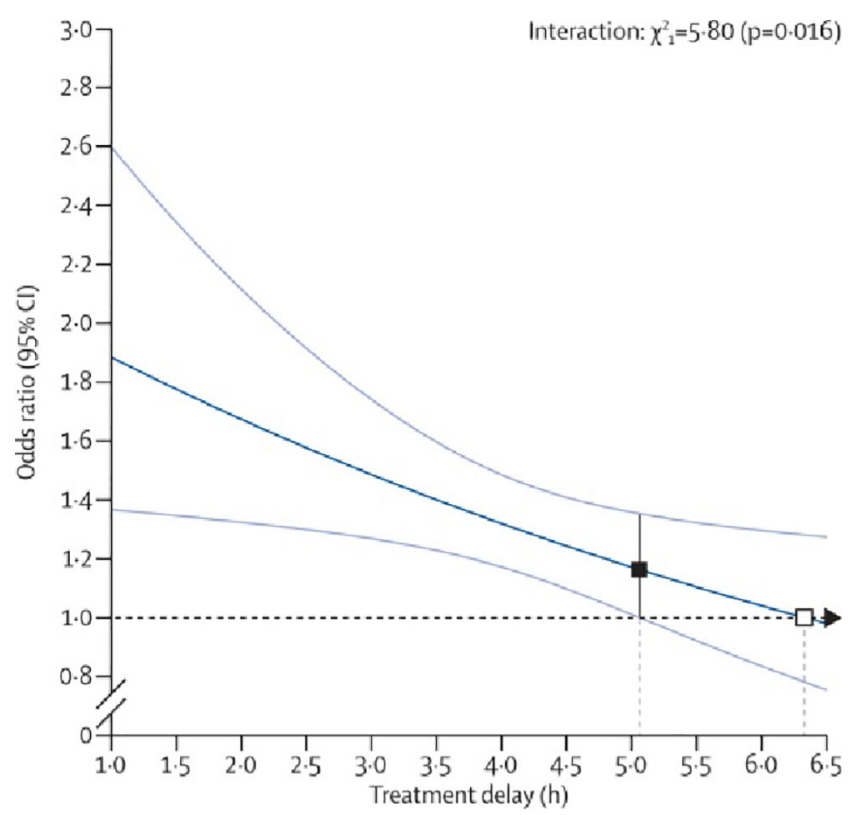

Fig. 1 Bénéfice du rt-PA en fonction du délai de traitement (d'après Hacke et al. [7]). En abscisse : délai (en heures) entre le début des symptômes et l'initiation du traitement. En ordonnée : (odds ratio [OR] et intervalle de confiance [IC] à $95 \%$ ) pour une évolution favorable (Rankin : 0 ou 1) à trois mois. Plus l'OR est élevé, plus le bénéfice est important. La borne inférieure de l'IC croise la ligne du 1 à 5 heures, ce qui signifie qu'il n’y a plus de bénéfice au-delà de 5 heures thérapeutiques. Ces traitements font l'objet de recommandations avec un haut niveau de preuves européennes et américaines $[10,11]$.

\section{Freins au parcours idéal des patients}

\section{Mauvaise orientation des patients}

Aujourd'hui, on considère qu'en France moins de $10 \%$ des patients ayant un IC reçoivent réellement le traitement fibrinolytique. Les causes les plus fréquemment avancées résident dans les délais très contraints, la méconnaissance par le grand public de la gravité des symptômes (seuls 30 \% identifient la faiblesse brutale de l'hémicorps comme un signe d'AVC) et de la conduite à tenir (seuls $50 \%$ des Français ont recours au Centre 15), le nombre trop faible de structures capables d'administrer le traitement et la crainte des complications hémorragiques.

\section{Morbidité et mortalité liées à la fibrinolyse}

La transformation hémorragique symptomatique est la complication redoutable du traitement fibrinolytique [12]. Plusieurs définitions de l'hémorragie intracérébrale symptomatique ont été proposées dans les essais sur le rt-PA. Selon la définition SITS-MOST (augmentation de quatre points sur score NIHSS), le taux d'hémorragies symptomatiques n'était que de $1,5 \%$. Selon la définition NINDS, il était de $7 \%$ à sept jours, et la mortalité à trois mois était de 11,8\% (contre $17,3 \%$ dans les essais thérapeutiques). Un âge moyen plus faible et une moindre sévérité des IC dans l'étude SITSMOST pourraient expliquer ce taux plus faible que celui observé dans les essais et dans d'autres registres. La mortalité était plus élevée dans les centres ayant une faible expérience de la thrombolyse, mais restait inférieure à celle observée dans les essais thérapeutiques, et le taux d'hémorragies symptomatiques n'était pas associé à l'expérience du centre. Il faut toutefois noter que seuls les centres volontaires ont participé à ce registre et qu'un audit n'a pu être réalisé que pour $10 \%$ des données environ.

Concernant le risque lié aux délais, il était important d'avoir des données sur le taux de complications hémorragiques rencontré dans la fenêtre 3-4,5 heures. En se fondant sur le registre SITS-ISTR (une extension du registre SITSMOST à d'autres pays), les patients traités au-delà de trois heures avaient un léger surrisque de complications hémorragiques et un pronostic un peu moins bon, mais la différence absolue entre les deux fenêtres était faible, confortant l'intérêt du traitement entre 3 et 4,5 heures [13].

Les principaux facteurs de risque d'hémorragie intracérébrale symptomatique sous rt-PA sont l'âge, la sévérité initiale, une pression artérielle élevée, un diabète et/ou une hyperglycémie, l'existence d'un œdème cérébral ou d'un 
effet de masse, un taux de plaquettes inférieur à 150 000/ $\mathrm{mm}^{3}$ et le non-respect des critères d'inclusion et d'exclusion. Alors que tout médicament antithrombotique est contreindiqué dans les 24 heures qui suivent une thrombolyse IV, un traitement antiplaquettaire préalablement à l'administration de rt-PA ne constitue pas une contre-indication, même s'il est associé à un très léger excès de risque de complications hémorragiques (essentiellement pour le clopidogrel).

L'efficacité de la thrombolyse IV varie en fonction de la localisation de l'occlusion artérielle. Le taux de recanalisation de l'artère occluse n'a pas été évalué dans les essais randomisés. Dans les études non contrôlées, le taux de recanalisation varie de 20 à $60 \%$, avec une « moyenne » estimée à $46 \%$ dans une revue systématique selon les études et la technique d'évaluation pour évaluer l'artère [15]. Le monitoring par angiographie conventionnelle des patients thrombolysés a montré qu'à 60 minutes après le début de la thrombolyse IV la recanalisation artérielle était obtenue chez:

- $8,7 \%$ des patients avec une occlusion de l'artère carotide interne (ACI) ;

- $35,1 \%$ des patients avec une occlusion proximale de l'artère cérébrale moyenne (ACM) ;

- $65,9 \%$ des patients avec une occlusion distale de l'ACM [14].

En résumé, plus l'occlusion est proximale, plus le taux de recanalisation décroît. Les patients avec une occlusion de l'ACI et/ou de l'ACM proximale sont donc de « mauvais répondeurs » à la thrombolyse IV. Ce sont justement ces occlusions proximales qui sont les plus grands pourvoyeurs de handicap et de mortalité. Cela souligne l'importance de développer de nouvelles stratégies pour augmenter ce taux de recanalisation.

Ces limites de la thrombolyse IV par altéplase ont fait développer d'autres techniques de revascularisation comme la thrombectomie (cf. paragraphe ci-dessous), et d'autres thrombolytiques sont en cours d'étude.

\section{Thrombolyse IV par le ténectéplase}

Le ténectéplase, thrombolytique privilégié dans l'infarctus du myocarde, pourrait dans les années à venir être une alternative potentielle du rt-PA. Sa demi-vie plus longue (une à trois heures versus quatre à cinq minutes) permet une facilité d'administration à la phase aiguë sous forme de bolus unique. L'étude ATTEST, comparant la thrombolyse par altéplase versus ténectéplase à la dose de $0,25 \mathrm{mg} / \mathrm{kg}$, n'avait retrouvé aucune différence sur le $\mathrm{mRS}$ à trois mois et la pénombre sauvée en scanner de perfusion [16]. Le nombre de complications hémorragiques cérébrales était similaire. Plus récemment, l'étude NOR-TEST a conclu à une nonsupériorité du ténectéplase à la dose de $0,4 \mathrm{mg} / \mathrm{kg}$ comparé à l'altéplase et à une sécurité d'utilisation identique (taux d'hématomes comparable) [17]. Il est à noter toutefois que les patients étudiés avaient des IC de faible gravité (NIHSS médian à 5,6). D'autres études sont en cours pour préciser la place du ténectéplase (EXTEND-IA-TK, TASTE, ATTEST, TEMPO) et notamment avant la thrombectomie.

\section{Thrombectomie mécanique}

Jusqu'en 2015, le traitement endovasculaire était une technique de revascularisation en évaluation. Trois essais, publiés en 2013, IMS III (the Interventional Management of Stroke III) [18], MR RESCUE (Magnetic Resonance and Recanalization of Stroke Clots Using Embolectomy) [19], SYNTHESIS expansion (Intra-arterial versus Systemic Thrombolysis for Acute Ischemic Stroke) [20] n'avaient pas montré de supériorité de l'approche endovasculaire par rapport à la thrombolyse IV. L'année 2015 a marqué un tournant avec cinq essais thérapeutiques qui ont prouvé l'efficacité de la thrombectomie mécanique (TM) en association avec la thrombolyse IV dans le traitement des IC avec occlusion des artères de gros calibre de la circulation antérieure (ACI ; ACM) [21-25].

Une meilleure sélection des patients et l'arrivée de nouveaux dispositifs expliquent en partie ces différences de résultats. La technique de référence se tourne aujourd'hui vers des stents non largables (stent retriever) qui permettent de capturer le caillot après l'impaction du thrombus contre la paroi (stent déployé), puis de retirer le thrombus à l'aide du stent (sans que le stent soit largué).

C'est l'étude randomisée néerlandaise MR CLEAN [21] (Multicenter Randomized Clinical trial of Endovascular Treatment in the Netherlands), publiée en janvier 2015, qui a, pour la première fois, confirmé l'approche avec une stratégie combinée associant une thrombolyse IV et une TM. Il s'agissait de patients de plus de 18 ans, avec un IC sur occlusion proximale de la circulation antérieure et un score NIHSS supérieur ou égal à 2. La TM était réalisée dans les six heures après le début des symptômes. Le critère de jugement principal était le pourcentage de bon pronostic à trois mois (défini par un mRS $<2$ ). À trois mois, 32,6\% des patients du groupe thrombolyse IV + TM ont eu une évolution favorable versus $19,1 \%$ dans le groupe thrombolyse IV seule (OR : 1,67 ; IC $95 \%=[1,2-2,3])$. À la suite de la positivité de MR CLEAN, quatre autres études ont été interrompues sur la base d'analyses intermédiaires ou perte d'équipoise devant la supériorité rapportée de la TM.

L'étude ESCAPE [22] (Endovascular treatment for Small Core and Anterior circulation Proximal with Emphasis on minimizing $\mathrm{CT}$ to recanalization times) a été prématurément arrêtée en raison d'une analyse intermédiaire positive. Trois cent seize patients, avec un score NIHSS supérieur à 5 , de bonnes collatérales et un IC de petite taille défini par un 
score ASPECTS (score radiologique évaluant la taille de l'IC (Fig. 2)) supérieur à 5, ont été randomisés dans deux groupes : TM dans les 12 heures versus absence de TM. Une thrombolyse IV était administrée avant randomisation si les patients étaient éligibles à la thrombolyse IV ( $73 \%$ des patients). Dans le groupe TM, le temps moyen pour la revascularisation était de 84 minutes. À trois mois, $53 \%$ des patients du groupe TM ont eu une évolution favorable $(\mathrm{mRS} \leq 2)$ versus $29,3 \%$ dans le groupe témoin (OR : $2,6$; IC $95 \%=[2,0-4,7])$.

L'étude SWIFT-PRIME [23] (Solitaire With the Intention for Thrombectomy as Primary Endovascular Treatment) a également été prématurément arrêtée en raison d'une analyse intermédiaire positive. Cent quatre-vingt-seize patients, avec

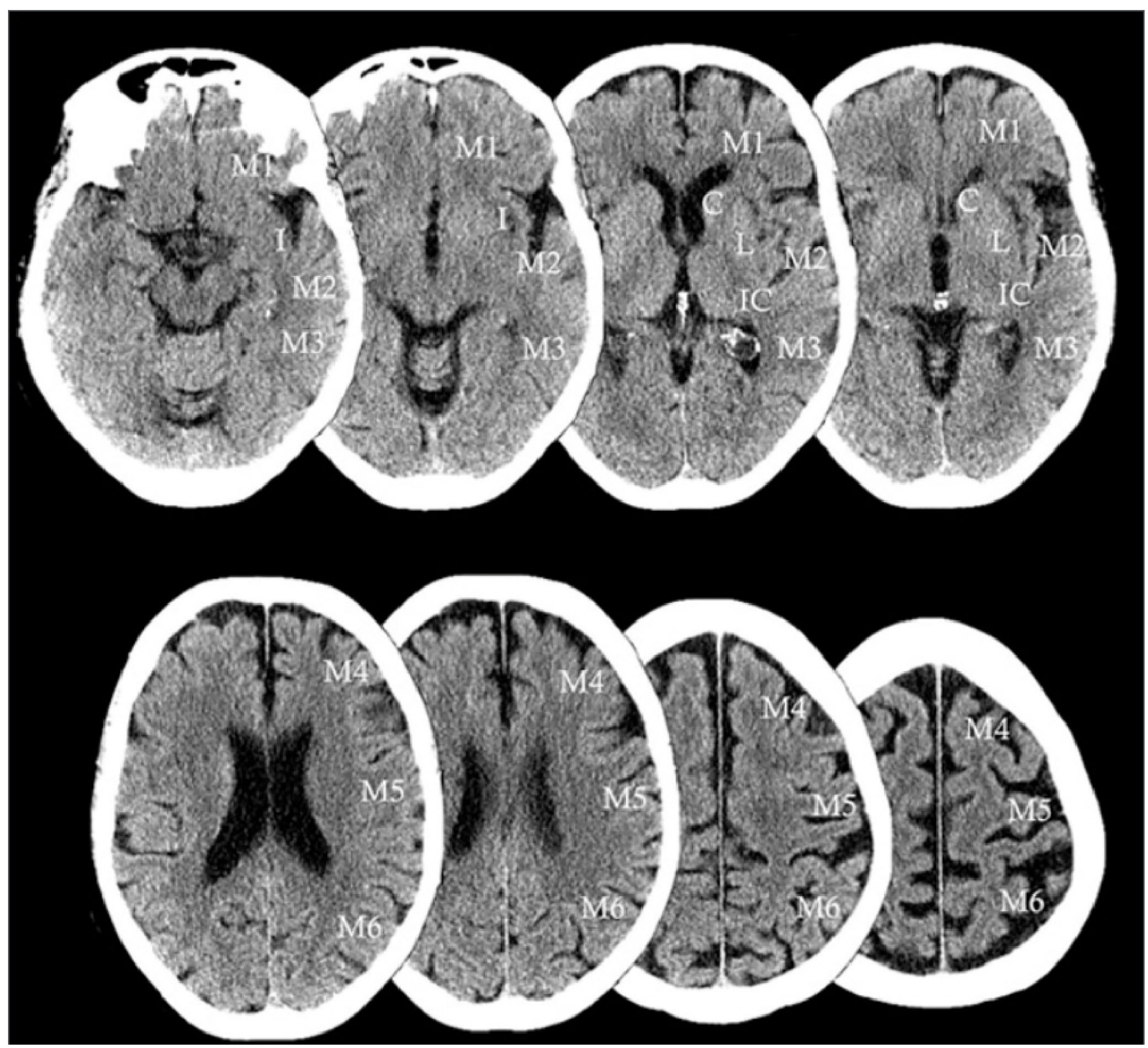

Fig. 2 Score ASPECT : score de dix points permettant d'évaluer les AVC ischémiques dans le territoire de l'artère cérébrale moyenne. Le territoire de l'artère cérébrale moyenne (ACM) est divisé en dix secteurs, sur une TDM sans injection en coupe axiale : régions profondes ou sous-corticales $(n=3)$ et régions superficielles ou corticales $(n=7): 1)$ C- noyau caudé ; 2) I- ruban insulaire ; 3) IC- Capsule interne ; 4) L- noyau lenticulaire ; 5) M1- ACM antérieure ; 6) M2- territoire de l'ACM devant le ruban insulaire ; 7) M3- ACM postérieure ; 8) M4- territoire ACM antérosupérieure ; 9) M5- territoire ACM latérosupérieure ; 10) M6- territoire ACM postéroinférieure. Le score est obtenu après examen de chacune de ces zones. En absence d'hypodensité visible (zone normale), on compte un point. Un score à 10 signifie donc une absence totale d'hypodensité visible. Un score à 0 : hypodensité de tout le territoire de l'ACM. Les hypodensités doivent être visibles sur au moins deux coupes différentes adjacentes Source : http://www.aspectsinstroke.com/ 
une occlusion de la circulation antérieure proximale, un score NIHSS entre 8 et 29, un score ASPECTS supérieur à 6 , une hypodensité au scanner inférieure à un tiers du territoire sylvien et traités par thrombolyse IV dans les 4 heures 30 minutes, ont été randomisés en deux groupes : TM dans les six heures versus absence de TM. À trois mois, $60 \%$ des patients du groupe TM ont eu une évolution favorable $(\mathrm{mRS} \leq 2)$ dans le groupe TM versus $35 \%$ dans le groupe témoin $(p=0,01, \mathrm{NNT}=4)$.

Dans EXTEND-IA [24] (Extending the Time for Thrombolysis in Emergency Neurological Deficits with IntraArterial therapy), 70 patients ont été randomisés. Pour être inclus, les patients devaient avoir un IC par occlusion artérielle proximale de la circulation antérieure, avoir été traités par thrombolyse IV dans les 4 heures 30 minutes et avoir un mismatch documenté (IC de petite taille associé à une large hypoperfusion sur l'imagerie de perfusion). Le geste de TM devait être réalisé dans les six heures. Le taux d'évolution favorable a été de $71 \%$ dans le groupe TM versus $40 \%$ dans le groupe de thrombolyse IV.

REVASCAT [25] (Randomized Trial of Revascularization with Solitaire FR Device versus Best Medical Therapy in the Treatment of Acute Stroke Due to Anterior Circulation Large Vessel Occlusion Presenting within Eight Hours of Symptom Onset) a inclus 206 patients, âgés de 18 à 80 ans, avec une occlusion proximale de la circulation antérieure, un IC de petite taille (score ASPECTS $\geq 7$ sur scanner cérébral et $\geq 6$ sur l'IRM cérébrale) et avec un score NIHSS supérieur ou égal à 6 , pouvant être traités dans les huit heures. Les patients devaient avoir reçu la thrombolyse IV dans les 4 heures 30 minutes, sans revascularisation, ou devaient avoir une contre-indication à la thrombolyse IV pour être inclus. Cette étude a été interrompue par défaut d'inclusion lié aux résultats des études précédentes. À trois mois, 43,7\% des patients du groupe TM ont eu une évolution favorable $(\mathrm{mRS} \leq 2)$ versus $28,2 \%$ dans le groupe témoin (OR : 2,1 ; IC $95 \%=[1,1-4,0])$.

Dans les cinq études précédemment citées, il n'y avait pas de différence dans la survenue d'hémorragie intracrânienne entre les groupes TM et témoin. Seule l'étude ESCAPE a montré une réduction de la mortalité dans le groupe TM versus le groupe témoin $(10,4$ versus $19 \%, p=0,04)$.

Au vu de ces différentes études, il est important de noter :

- les études ont porté sur un faible nombre de patients ;

- la majorité des patients traités dans le groupe TM ont reçu une thrombolyse IV (entre 71 et $100 \%$ ). Les analyses en sous-groupes suggèrent que la TM est, également, efficace en l'absence de thrombolyse IV;

- le délai d'administration de la thrombolyse IV a été particulièrement court dans ces études : entre 87 minutes (MR CLEAN) et 127 minutes (EXTEND-IA) dans le groupe TM ; et entre 85 minutes (MR CLEAN) et 145 minutes (EXTEND-IA) dans le groupe témoin ;

- l'hétérogénéité des critères d'inclusion cliniques (score NIHSS), en imagerie (évaluation de la zone de pénombre, score ASPECTS, collatéralités, etc.) et de la fenêtre thérapeutique. Il faut préciser que même si, dans certaines études, les patients pouvaient être revascularisés jusqu'à 12 heures après le début des symptômes, la très large majorité l'a été dans les six heures (plus de $80 \%$ ) ;

- l'âge ne doit pas être un critère d'exclusion à la TM : les analyses en sous-groupes suggèrent un bénéfice de la TM chez les patients âgés de plus de 80 ans.

La méta-analyse de Saver et al. à partir de ces différentes études confirme une efficacité du traitement jusqu'à 7,3 heures [26] (Fig. 3).

À ce jour, conformément aux recommandations européennes [27], la thrombolyse IV doit être réalisée dans les 4 heures 30 minutes, sans retarder la thrombectomie qui doit être réalisée dans les six heures, lors d'occlusion proximale.

Tout récemment, l'étude DAWN [28] (DWI Assessment with clinical mismatch in the triage of Wake-up and mate presenting strokes undergoing Neurointervention) avait comme objectif de démontrer la supériorité de la thrombectomie (par stent retriever TREVO) sur le traitement médical chez des patients très sélectionnés avec occlusion antérieure proximale et mismatch radioclinique, traités entre 6 et 24 heures. Les critères d'évaluation étaient un mRS moyen et mRS 0-2 à trois mois. Deux étaient différenciés : 6-12 heures/12-24 heures. L'étude a été arrêtée après analyse intermédiaire devant la supériorité de la TM. Au total,

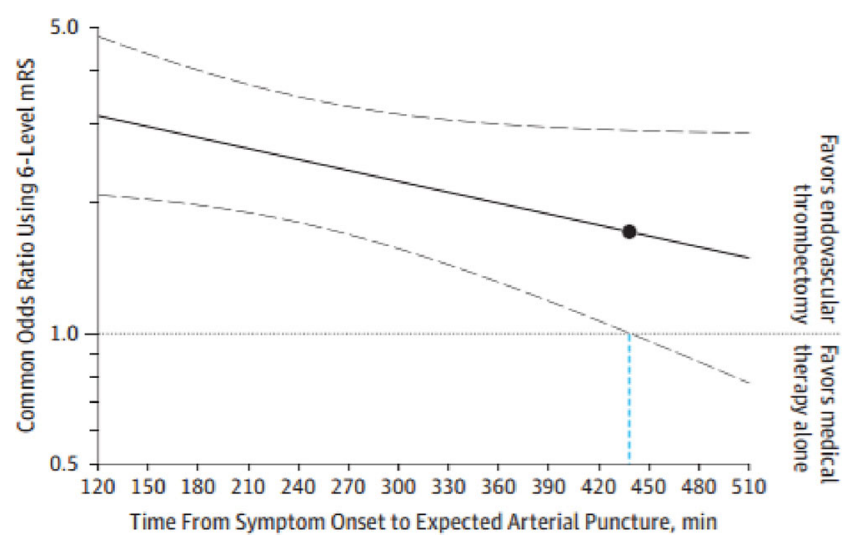

Fig. 3 Bénéfice de la thrombectomie en fonction du délai de traitement (d'après Jovin et al. [25]). En abscisse : délai (en minutes) entre le début des symptômes et la ponction. En ordonnée : (odds ratio [OR] et intervalle de confiance [IC] à $95 \%$ ) pour une évolution favorable (Rankin : 0 ou 1) à trois mois. Plus l'OR est élevé, plus le bénéfice est important. La borne inférieure de l'IC croise la ligne du 1 à 438 minutes, ce qui signifie qu'il n'y a plus de bénéfice audelà de 7,3 heures 
206 patients ont été inclus : 107 dans le groupe thrombectomie et 99 dans le groupe témoin. Le score de Rankin pondéré à trois mois était de 5,5 dans le groupe thrombectomisé et 3,4 dans le groupe témoin (IC $95 \%=[1,1-3,0]$ ), et le taux de patients autonomes à trois mois était de $49 \%$ dans le groupe thrombectomie contre $13 \%$ dans le groupe témoin (IC $95 \%=[24-44])$. Les taux de complications hémorragiques et de décès étaient comparables dans les deux groupes. Il faut néanmoins retenir que parmi les patients inclus dans le groupe thrombectomie, seuls dix d'entre eux avaient une heure de début certaine avec témoin oculaire et que les autres patients étaient essentiellement des AVC du réveil (67\%).

Ces données doivent être confirmées par d'autres études. Les résultats de l'étude DEFUSE III sont en attente.

La figure 4 résume les stratégies de revascularisation des IC en phase aiguë selon le délai.

\section{Impact organisationnel : le parcours du patient}

Le parcours de soins du patient se complexifie avec ces nouvelles données démontrant le bénéfice de la thrombectomie. Aujourd'hui, s'il existe 134 UNV réparties inégalement sur le territoire, seulement 37 d'entre elles sont attenantes à un service de NRI capable de pratiquer une thrombectomie.

Dès l'appel à la régulation du Samu pour un patient suspect d'AVC se posent trois questions : - le diagnostic d'AVC est-il certain ? - S'agit-il d'une ischémie ou d'une hémorragie ? Dans le cas d'un IC existe-t-il une occlusion intracrânienne ? L'imagerie conditionne le parcours patient et sa prise en charge thérapeutique. Depuis les trois dernières décennies est apparu le concept de drip en ship et de mother ship [29]. Faut-il adresser directement (mother ship) le patient dans une UNV de recours avec centre de NRI ou le diriger vers un SAU en lien avec une UNV (par télémédecine) ou vers une UNV de proximité (sans NRI) afin de faire un diagnostic rapidement et de transférer le patient dans un second temps seulement si nécessaire (drip en ship) ? Le registre prospectif STRATIS a comparé les différentes stratégies d'orientation, à partir de 984 patients victimes d'AVC ischémiques de la circulation antérieure avec occlusion proximale et NIHSS $\geq 8$, thrombectomisés dans les moins de huit heures. Dans cette étude, $52 \%$ des patients dans le bras drip and ship avaient un mRS à trois mois entre 0 et 2 contre $60 \%$ des patients dans le bras mother ship $(p=0,02)$. La différence de pronostic entre les deux groupes n'est expliquée que par la réduction du délai symptômes-reperfusion [30]. Holodinsky et al. proposent une modélisation des deux parcours de soins, tenant compte notamment des distances entre l'UNV de proximité et l'UNV recours et les délais intrahospitaliers (délais heure d'arrivée-heure de thrombolyse, heure d'arrivée-ponction) [31].

Nogueira et al. rapportent une application smartphone d'aide au parcours du patient tenant compte des distances entre UNV de proximité et centre NRI, des délais intrahospitaliers, du délai de prise en charge, de la circulation en temps réel, etc. [32].

Plusieurs scores préhospitaliers de prédiction de l'occlusion artérielle, presque uniquement fondés sur des items du

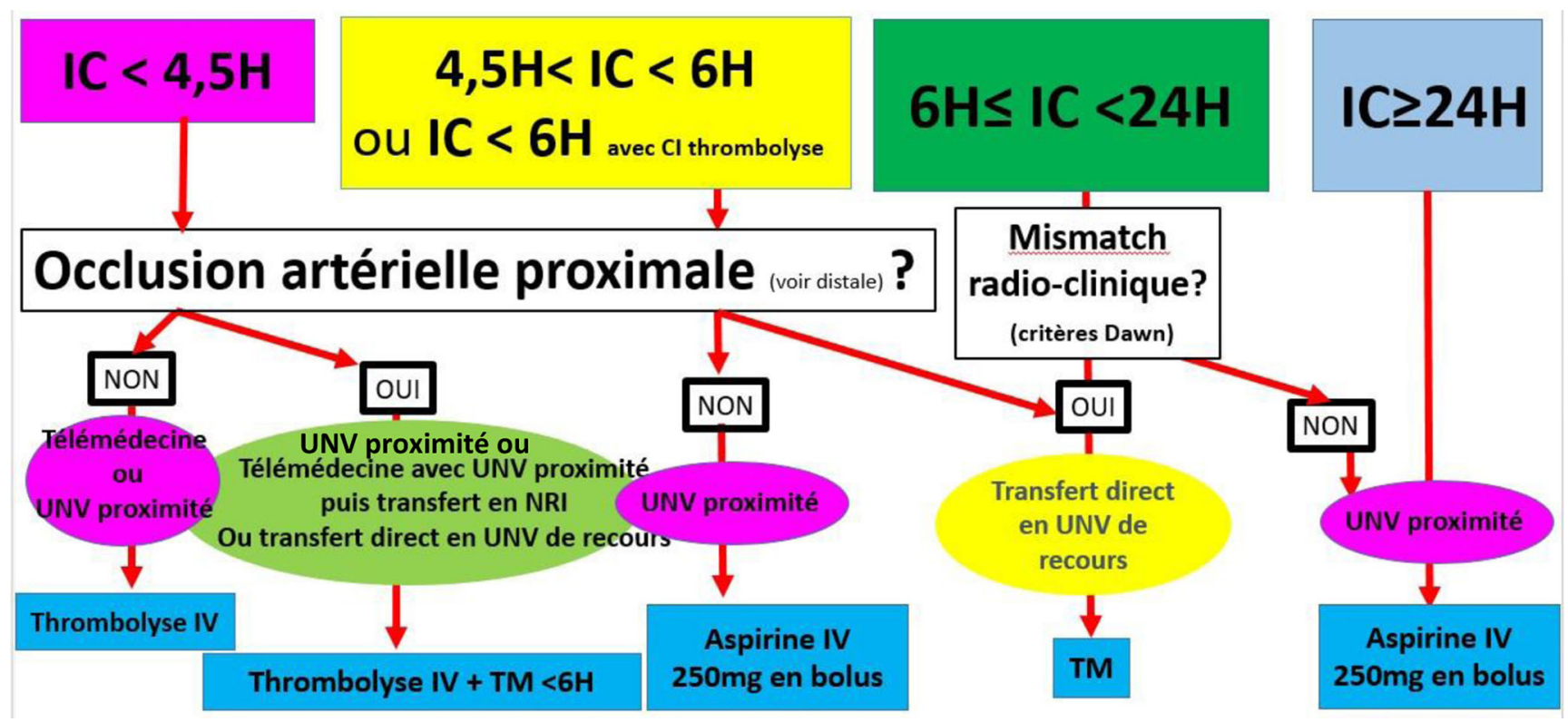

Fig. 4 Stratégies de revascularisation des infarctus cérébraux en phase aiguë selon le délai IC : infarctus cérébral ; H : délai par rapport au début des symptômes en heures ; TM : thrombectomie mécanique ; UNV proximité : établissement ayant une UNV sans service de neuroradiologie interventionnelle (NRI) ; UNV de recours : établissement ayant une UNV et disposant d'un service de NRI 
NIHSS, apparaissent ces dernières années (RACE, FAST ED, C-STAT, PASS, G-FAST, LAMS) [33-35]. Le score NIHSS reste le gold standard pour prédire l'occlusion proximale [36,37] mais est difficile d'utilisation en préhospitalier (complexe, chronophage, nécessité d'être formé). Quoi qu'il en soit, aucun score de prédilection de l'occlusion proximale ne remplacera l'imagerie artérielle qui intégrera peut-être demain les camions Samu, comme l'expérimentation allemande STEMO [38].

\section{Conclusion}

La prise en charge thérapeutique des patients victimes d'un AVC a connu, au cours de ces dernières années, une évolution majeure impactant l'organisation de la filière de soins. Chaque patient suspect d'AVC devrait bénéficier d'une expertise neurovasculaire urgente pour décider de l'attitude thérapeutique au plus vite. La présence d'une occlusion intracrânienne conditionne la prise en charge. La thrombolyse IV par altéplase doit être réalisée le plus tôt possible dans les 4 heures 30 minutes (idéalement dans les 90 minutes), suivie d'une TM lors d'occlusion proximale dans les six heures, fenêtre qui sera probablement élargie au vu des données récentes, chez des patients ultrasélectionnés (mismatch radioclinique). Reste à démontrer le bénéfice de la thrombectomie pour les AIT mineurs ou AIT, les occlusions distales et à réévaluer la place de la thrombolyse dans ce contexte. Enfin, le parcours optimum des patients entre mother ship et drip and ship devra être précisé.

Liens d'intérêts : l'auteur déclare ne pas avoir de lien d'intérêt.

\section{Références}

1. Circulaire DHOS/DGS/DGAS n ${ }^{\circ} 2003-517$ du 3 novembre 2003 relative à la prise en charge des accidents vasculaires cérébraux

2. Circulaire DHOS/O4/2007/108 du 22 mars 2007 relative à la place des unités neurovasculaires dans la prise en charge des patients présentant un accident vasculaire cérébral. NOR : SANH0730171C

3. Circulaire $\mathrm{DGOS} / \mathrm{R} 4 / \mathrm{R} 3 / \mathrm{PF} 3 / 2012 / 106$ du 6 mars 2012 relative à l'organisation des filières régionales de prise en charge des patients victimes d'accident vasculaire cérébral (AVC). NOR : ETSH1206895C. Disponible sur :a http://circulaire.legifrance. gouv.fr/pdf/2012/03/cir_34866.pdf

4. Stroke unit trialists' collaboration, (2007) Organised inpatient (stroke unit) care for stroke. Cochrane Database Syst Rev 4: CD000197

5. Seenan P, Long M, Langhorne P, (2007) Stroke units in their natural habitat: systematic review of observational studies. Stroke 38: $1886-1892$

6. The National Institute of Neurological Disorders and Stroke, rtPa Stroke Study Group, (1995) Tissue plasminogen activator for acute ischemic stroke. N Engl J Med 333: 1581-1587

7. Hacke W, Kaste M, Bluhmki E, Brozman M, Dávalos A, Guidetti D, Larrue V, Lees KR, Medeghri Z, Machnig T, Schneider D, von Kummer R, Wahlgren N, Toni D; ECASS Investigators, (2008) Thrombolysis with alteplase 3 to 4,5 hours after acute ischemic stroke. N Engl J Med 359: 1317-1329

8. Emberson J, Lees KR, Lyden P, Blackwell L, Albers G, Bluhmki E, Brott T, Cohen G, Davis S, Donnan G, Grotta J, Howard G, Kaste M, Koga M, von Kummer R, Lansberg M, Lindley RI, Murray G, Olivot JM, Parsons M, Tilley B, Toni D, Toyoda K, Wahlgren N, Wardlaw J, Whiteley W, del Zoppo GJ, Baigent C, Sandercock P, Hacke W; Stroke Thrombolysis Trialists' Collaborative Group, (2014) Effect of treatment delay, age, and stroke severity on the effects of intravenous thrombolysis with alteplase for acute ischaemic stroke: a meta-analysis of individual patient data from randomised trials. Lancet 384: 1929-1935

9. Wahlgren N, Ahmed N, Dávalos A, Ford GA, Grond M, Hacke W, Hennerici MG, Kaste M, Kuelkens S, Larrue V, Lees KR, Roine RO, Soinne L, Toni D, Vanhooren G; SITS-MOST investigators, (2007) Thrombolysis with alteplase for acute ischaemic stroke in the safe implementation of thrombolysis in stroke-monitoring study (sits-most): an observational study. Lancet 369: 275-282

10. Jauch EC, Saver JL, Adams HP Jr, Bruno A, Connors JJ, Demaerschalk BM, Khatri P, McMullan PW Jr, Qureshi AI, Rosenfield K, Scott PA, Summers DR, Wang DZ, Wintermark M, Yonas H; American Heart Association Stroke Council; Council on Cardiovascular Nursing; Council on Peripheral Vascular Disease; Council on Clinical Cardiology, (2013) Guidelines for the early management of patients with acute ischemic stroke: a guideline for healthcare professionals from the American Heart Association/American Stroke Association. Stroke 44: 870-947

11. The European Stroke Organisation (ESO) executive committee and the ESO Writing Committee, (2008) Guidelines for management of ischaemic stroke and transient ischaemic attack 2008. Cerebrovasc Dis 25: 457-507

12. Derex L, Nighoghossian N, (2008) Intracerebral haemorrhage after thrombolysis for acute ischaemic stroke: an update. J Neurol Neurosurg Psychiatry 79: 1093-1099

13. Ahmed N, Wahlgren N, Grond M, Hennerici M, Lees KR, Mikulik R, Parsons M, Roine RO, Toni D, Ringleb P, SITS investigators, (2010) Implementation and outcome of thrombolysis with alteplase 3-4.5 h after an acute stroke: an updated analysis from sits-istr. Lancet Neurol 9: 866-874

14. del Zoppo GJ1, Poeck K, Pessin MS, Wolpert SM, Furlan AJ, Ferbert A, Alberts MJ, Zivin JA, Wechsler L, Busse O, Greenlee R, Brass L, Mohr JP, Feldmann E, Hacke W, Kase C, Biller J, Gress D, Otis S (1992) Recombinant tissue plasminogen activator in acute thrombotic and embolic stroke. Ann Neurol 32: 78-86

15. Rha JH, Saver JL, (2007) The impact of recanalization on ischemic stroke outcome: a meta-analysis. Stroke 38: 967-973

16. Huang X, Cheripelli BK, Lloyd SM, Kalladka D, Moreton FC, Siddiqui A, Ford I, Muir KW, (2015) Alteplase versus tenecteplase for thrombolysis after ischaemic stroke (ATTEST): a phase 2, randomised, open-label, blinded endpoint study. Lancet Neurol 14: 368-376

17. Logallo N, Novotny V, Assmus J, Kvistad CE, Alteheld L, Rønning OM, Thommessen B, Amthor KF, Ihle-Hansen H, Kurz M, Tobro H, Kaur K, Stankiewicz M, Carlsson M, Morsund Å, Idicula T, Aamodt AH, Lund C, Næss H, WajeAndreassen U, Thomassen L, (2017) Tenecteplase versus alteplase for management of acute ischaemic stroke (NOR-TEST): a phase 3, randomised, open-label, blinded endpoint trial. Lancet Neurol 16: 781-788

18. Broderick JP, Palesch YY, Demchuk AM, Yeatts SD, Khatri P, Hill MD, Jauch EC, Jovin TG, Yan B, Silver FL, von Kummer R, Molina CA, Demaerschalk BM, Budzik R, Clark WM, Zaidat OO, Malisch TW, Goyal M, Schonewille WJ, Mazighi M, Engelter ST, Anderson C, Spilker J, Carrozzella J, Ryckborst KJ, Janis LS, Martin RH, Foster LD, Tomsick TA; Interventional Management of Stroke (IMS) III Investigators, (2013) Endovascular 
therapy after intravenous t-PA versus t-PA alone for stroke. N Engl J Med 368: 893-903

19. Kidwell CS, Jahan R, Gornbein J, Alger JR, Nenov V, Ajani Z, Feng L, Meyer BC, Olson S, Schwamm LH, Yoo AJ, Marshall RS, Meyers PM, Yavagal DR, Wintermark M, Guzy J, Starkman S, Saver JL, MR RESCUE Investigators, (2013) A trial of imaging selection and endovascular treatment for ischemic stroke. N Engl J Med 368: 914-923

20. Ciccone A, Valvassori L, Nichelatti M, Sgoifo A, Ponzio M, Sterzi R, Boccardi E; SYNTHESIS Expansion Investigators, (2013) Endovascular treatment for acute ischemic stroke. N Engl J Med 368:904-913

21. Berkhemer OA, Fransen PS, Beumer D, van den Berg LA, Lingsma HF, Yoo AJ, Schonewille WJ, Vos JA, Nederkoorn PJ, Wermer MJ, van Walderveen MA, Staals J, Hofmeijer J, van Oostayen JA, Lycklama à Nijeholt GJ, Boiten J, Brouwer PA, Emmer BJ, de Bruijn SF, van Dijk LC, Kappelle LJ, Lo RH, van Dijk EJ, de Vries J, de Kort PL, van Rooij WJ, van den Berg JS, van Hasselt BA, Aerden LA, Dallinga RJ, Visser MC, Bot JC, Vroomen PC, Eshghi O, Schreuder TH, Heijboer RJ, Keizer K, Tielbeek AV, den Hertog HM, Gerrits DG, van den Berg-Vos RM, Karas GB, Steyerberg EW, Flach HZ, Marquering HA, Sprengers ME, Jenniskens SF, Beenen LF, van den Berg R, Koudstaal PJ, van Zwam WH, Roos YB, van der Lugt A, van Oostenbrugge RJ, Majoie CB, Dippel DW, MR CLEAN Investigators, (2015) A randomized trial of intraarterial treatment for acute ischemic stroke. N Engl J Med 372: 11-20

22. Goyal M, Demchuk AM, Menon BK, Eesa M, Rempel JL, Thornton J, Roy D, Jovin TG, Willinsky RA, Sapkota BL, Dowlatshahi D, Frei DF, Kamal NR, Montanera WJ, Poppe AY, Ryckborst KJ, Silver FL, Shuaib A, Tampieri D, Williams D, Bang OY, Baxter BW, Burns PA, Choe H, Heo JH, Holmstedt CA, Jankowitz B, Kelly M, Linares G, Mandzia JL, Shankar J, Sohn SI, Swartz RH, Barber PA, Coutts SB, Smith EE, Morrish WF, Weill A, Subramaniam S, Mitha AP, Wong JH, Lowerison MW, Sajobi TT, Hill MD; ESCAPE Trial Investigators, (2015) Randomized assessment of rapid endovascular treatment of ischemic stroke. N Engl J Med 372: 1019-1030

23. Saver JL, Goyal M, Bonafe A, Diener HC, Levy EI, Pereira VM, Albers GW, Cognard C, Cohen DJ, Hacke W, Jansen O, Jovin TG, Mattle HP, Nogueira RG, Siddiqui AH, Yavagal DR, Baxter BW, Devlin TG, Lopes DK, Reddy VK, du Mesnil de Rochemont R, Singer OC, Jahan R; SWIFT PRIME Investigators, (2015) Stent-retriever thrombectomy after intravenous t-PA vs. tPA alone in stroke. N Engl J Med 372: 2285-2295

24. Campbell BC, Mitchell PJ, Kleinig TJ, Dewey HM, Churilov L, Yassi N, Yan B, Dowling RJ, Parsons MW, Oxley TJ, Wu TY, Brooks M, Simpson MA, Miteff F, Levi CR, Krause M, Harrington TJ, Faulder KC, Steinfort BS, Priglinger M, Ang T, Scroop R, Barber PA, McGuinness B, Wijeratne T, Phan TG, Chong W, Chandra RV, Bladin CF, Badve M, Rice H, de Villiers L, Ma H, Desmond PM, Donnan GA, Davis SM; EXTEND-IA Investigators, (2015) Endovascular therapy for ischemic stroke with perfusion-imaging selection. N Engl J Med 372: 1009-1018

25. Jovin TG, Chamorro A, Cobo E, de Miquel MA, Molina CA, Rovira A, San Román L, Serena J, Abilleira S, Ribó M, Millán M, Urra X, Cardona P, López-Cancio E, Tomasello A, Castaño C, Blasco J, Aja L, Dorado L, Quesada H, Rubiera M, HernandezPérez M, Goyal M, Demchuk AM, von Kummer R, Gallofré M, Dávalos A; REVASCAT Trial Investigators, (2015) Thrombectomy within 8 hours after symptom onset in ischemic stroke. N Engl J Med 372: 2296-2306

26. Saver JL, Goyal M, Hill MD; HERMES Collaborators, (2017) Time to endovascular thrombectomy for acute stroke-reply. JAMA 317: 1175-1176
27. Wahlgren N, Moreira T, Michel P, (2016) Mechanical thrombectomy in acute ischemic stroke: Consensus statement by ESOKarolinska Stroke Update 2014/2015, supported by ESO, ESMINT, ESNR and EAN. Int J Stroke 11: 134-147

28. Nogueira RG, Jadhav AP, Haussen DC, (2018) Thrombectomy 6 to 24 hours after stroke with a mismatch between deficit and infarct. N Engl J Med 378: 11-21

29. Daubail B, Ricolfi F, Thouant P, Vogue C, Chavent A, Osseby GV, Hervieu-Begue M, Delpont B, Mangola B, Bejot Y, Giroud M, (2016) Impact of mechanical thrombectomy on the organization of the management of acute ischemic stroke. Eur Neurol 75:41-47

30. Froehler MT, Saver JL, Zaidat OO, Jahan R, Aziz-Sultan MA, Klucznik RP, Haussen DC, Hellinger FR Jr, Yavagal DR, Yao TL, Liebeskind DS, Jadhav AP, Gupta R, Hassan AE, Martin CO, Bozorgchami H, Kaushal R, Nogueira RG, Gandhi RH, Peterson EC, Dashti SR, Given CA 2nd, Mehta BP, Deshmukh V, Starkman S, Linfante I, McPherson SH, Kvamme P, Grobelny TJ, Hussain MS, Thacker I, Vora N, Chen PR, Monteith SJ, Ecker RD, Schirmer CM, Sauvageau E, Abou-Chebl A, Derdeyn CP, Maidan L, Badruddin A, Siddiqui AH, Dumont TM, Alhajeri A, Taqi MA, Asi K, Carpenter J, Boulos A, Jindal G, Puri AS, Chitale R, Deshaies EM, Robinson DH, Kallmes DF, Baxter BW, Jumaa MA, Sunenshine P, Majjhoo A, English JD, Suzuki S, Fessler RD, Delgado Almandoz JE, Martin JC, Mueller-Kronast NH; STRATIS Investigators, (2017) Interhospital transfer before thrombectomy is associated with delayed treatment and worse outcome in the STRATIS Registry (Systematic Evaluation of Patients Treated With Neurothrombectomy Devices for Acute Ischemic Stroke). Circulation 136: 2311-2321

31. Holodinsky JK, Williamson TS, Kamal N, Mayank D, Hill MD, Goyal M, (2017) Drip and ship versus direct to comprehensive stroke center: conditional probability modeling. Stroke 48: 233-238

32. Nogueira RG, Silva GS, Lima FO, Yeh YC, Fleming C, Branco D, Yancey AH, Ratcliff JJ, Wages RK, Doss E, Bouslama M, Grossberg JA, Haussen DC, Sakano T, Frankel MR, (2017) The FAST-ED App: a smartphone platform for the field triage of patients with stroke. Stroke 48: 1278-1284

33. Pérez de la Ossa N, Carrera D, Gorchs M, Querol M, Millán M, Gomis M, Dorado L, López-Cancio E, Hernández-Pérez M, Chicharro V, Escalada X, Jiménez X, Dávalos A, (2014) Design and validation of a prehospital stroke scale to predict large arterial occlusion: the rapid arterial occlusion evaluation scale. Stroke 45: 87-91

34. Lima FO, Silva GS, Furie KL, Frankel MR, Lev MH, Camargo ÉC, Haussen DC, Singhal AB, Koroshetz WJ, Smith WS, Nogueira RG, (2016) Field assessment stroke triage for emergency destination: a simple and accurate prehospital scale to detect large vessel occlusion strokes. Stroke 47: 1997-2002

35. Heldner MR, Mattle HP, Fischer U, (2016) Clinical prediction of large vessel occlusion in anterior circulation stroke: mission impossible? J Neurol 263: 1633-1640

36. Zhao H, Coote S, Pesavento L, Churilov L, Dewey HM, Davis SM, Campbell BC, (2017) Large vessel occlusion scales increase delivery to endovascular centers without excessive harm from misclassifications. Stroke 48: 568-573

37. Turc G, Maïer B, Naggara O, Seners $P$, Isabel C, Tisserand M, Raynouard I, Edjlali M, Calvet D, Baron JC, Mas JL, Oppenheim C, (2016) Clinical scales do not reliably identify acute ischemic stroke patients with large-artery occlusion. Stroke 47: 1466-1472

38. Ebinger M, Winter B, Wendt M, Weber JE, Waldschmidt C, Rozanski M, Kunz A, Koch P, Kellner PA, Gierhake D, Villringer K, Fiebach JB, Grittner U, Hartmann A, Mackert BM, Endres M, Audebert HJ; STEMO Consortium, (2014) Effect of the use of ambulance-based thrombolysis on time to thrombolysis in acute ischemic stroke: a randomized clinical trial. JAMA 311: $1622-1631$ 